\title{
ON A PERTURBED CONSERVATIVE SYSTEM OF SEMILINEAR WAVE EQUATIONS WITH PERIODIC-DIRICHLET BOUNDARY CONDITIONS
}

\author{
JINHAI CHEN ${ }^{\bowtie}$ and DONAL O'REGAN
}

(Received 16 June 2009)

\begin{abstract}
In this paper, some existence and uniqueness results for generalized solutions to a periodic-Dirichlet problem for semilinear wave equations are given, using a global inverse function theorem. These results extend those known in the literature.
\end{abstract}

2000 Mathematics subject classification: primary 35B10, 35D05, 35L05, 35L20; secondary 35P05, 47F05.

Keywords and phrases: wave equation, periodic-Dirichlet problem, generalized solution, unique existence, global inverse function theorem.

\section{Introduction}

Let $\mathcal{J}=[0,2 \pi] \times[0, \pi]$, let $n \geq 1$ be an integer, let $\mathbb{N}^{*}$ be the set of nonnegative integers, and let $F: \mathcal{J} \times \mathbb{R}^{n} \rightarrow \mathbb{R}^{n}$ be a function of class $C^{2}$. Suppose that $V$ : $\mathcal{J} \times \mathbb{R}^{n} \rightarrow \mathbb{R}$ is a function of class $C^{2}$ whose gradient and Hessian matrix with respect to $u$ are denoted by $V^{\prime}$ and $V^{\prime \prime}$, respectively. Let $h \in \mathcal{H}$ with $\mathcal{H}=\left(L^{2}(\mathcal{J})\right)^{n}$ be given, with the usual inner product $\langle\cdot, \cdot\rangle$ and corresponding norm $\|\cdot\|$. We consider the system of semilinear wave equations

$$
u_{t t}-u_{x x}-V^{\prime}(t, x, u)+F(t, x, u)=h(t, x),
$$

where subscripts denote the partial derivative, and where $F(t, x, u)$ is called a perturbing term. By a generalized solution of the periodic-Dirichlet problem on $\mathcal{J}$ for (1.1) (or GPDS on $\mathcal{J}$ for short) we mean an element $u \in \mathcal{H}$ such that

$$
\left\langle u, v_{t t}-v_{x x}\right\rangle-\left\langle v, V^{\prime}(t, x, u)\right\rangle+\langle v, F(t, x, u)\rangle=\langle h(t, x), v\rangle,
$$

for all $v \in\left(C^{2}(\mathcal{J})\right)^{n}$ satisfying

$$
\begin{gathered}
v(t, 0)=v(t, \pi)=0, \quad \forall t \in[0,2 \pi] ; \\
v(0, x)=v(2 \pi, x), \quad v_{t}(0, x)=v_{t}(2 \pi, x), \quad \forall x \in[0, \pi] .
\end{gathered}
$$

(C) 2010 Australian Mathematical Publishing Association Inc. 0004-9727/2010 \$16.00 
When the perturbing term $F(t, x, u)$ is 0 , it is easy to see that the conservative system

$$
u_{t t}-u_{x x}-V^{\prime}(u)=h(t, x),
$$

is included in the system (1.1). In [6], Mawhin obtained the following existence and uniqueness theorem for the GPDS of (1.2) on $\mathcal{J}$ using a Galerkin type argument similar to that in Bates and Castro [2] and a global inverse function theorem.

TheOREM 1.1. Let $V: \mathbb{R}^{n} \rightarrow \mathbb{R}$ be a function of class $C^{2}$ and let $\mathcal{J}=[0,2 \pi] \times$ $[0, \pi]$. Assume that there exist two $n \times n$ symmetric matrices $A$ and $B$, with respective eigenvalues $\alpha_{1} \leq \alpha_{2} \leq \cdots \leq \alpha_{n}$ and $\beta_{1} \leq \beta_{2} \leq \cdots \leq \beta_{n}$, such that

$$
A \leq V^{\prime \prime}(u) \leq B
$$

for every $u \in \mathbb{R}^{n}$ and

$$
\bigcup_{k=1}^{n}\left[\alpha_{k}, \beta_{k}\right] \cap\left\{m^{2}-l^{2} \mid l \in \mathbb{Z}, m \in \mathbb{N}^{*}\right\}=\varnothing .
$$

Then (1.2) with the periodic-Dirichlet boundary conditions on $\mathcal{J}$ has a unique generalized solution $u \in\left(L^{2}(\mathcal{J})\right)^{n}$ for every $h \in\left(L^{2}(\mathcal{J})\right)^{n}$.

For more results on the existence of GPDS on $\mathcal{J}$ of (1.1), we refer the reader to $[1,4,5,7]$ and the references therein.

In this paper, we establish some new sufficient conditions for the existence of a unique GPDS on $\mathcal{J}$ of (1.1). Our proof is different from those mentioned above, and we use a new global inverse function theorem. Our results extend those in [1, 2, 4-7].

Throughout this paper we use the following assumption.

(A1). The eigenvalues $\lambda_{i}\left(V^{\prime \prime}(t, x, u)\right), i=1, \ldots, n$, of $V^{\prime \prime}(t, x, u)$ satisfy

$$
\alpha_{i}+\phi_{i}(t, x,\|u\|) \leq \lambda_{i}\left(V^{\prime \prime}\right) \leq \beta_{i}-\varphi_{i}(t, x,\|u\|)
$$

on $\mathcal{J} \times \mathbb{R}^{n}$, where $\alpha_{i}, \beta_{i} \in\left\{m^{2}-l^{2} \mid l \in \mathbb{Z}, m \in \mathbb{N}^{*}\right\}, i=1, \ldots, n$, are consecutive, $\phi_{i}(t, x, s)$ and $\varphi_{i}(t, x, s), i=1, \ldots, n$, are continuous functions defined from $\mathcal{J} \times$ $[0, \infty)$ to $(0, \infty)$, they are nonincreasing with respect to $s$, and

$$
\int_{0}^{+\infty} \min _{1 \leq i \leq n,(t, x) \in \mathcal{J}}\left\{\phi_{i}(t, x, s), \varphi_{i}(t, x, s)\right\} d s=+\infty .
$$

Here we say that $\alpha_{i}, \beta_{i} \in\left\{m^{2}-l^{2} \mid l \in \mathbb{Z}, m \in \mathbb{N}^{*}\right\}$ are consecutive, for $i=1, \ldots, n$, if

$$
\bigcup_{j=1}^{n}\left(\alpha_{i}, \beta_{i}\right) \cap\left\{m^{2}-l^{2} \mid l \in \mathbb{Z}, m \in \mathbb{N}^{*}\right\}=\emptyset,
$$

and $\alpha_{1} \leq \alpha_{2} \leq \cdots \leq \alpha_{n}, \beta_{1} \leq \beta_{2} \leq \cdots \leq \beta_{n}, \alpha_{i}<\beta_{i}$ for each $i$. 


\section{Abstract reformulation}

If $\left\{c_{k} \mid 1 \leq k \leq n\right\}$ denotes an orthonormal basis in $\mathbb{R}^{n}$ and if we set

$$
v_{l m}(t, x)=\exp (i l t) \sin m x, \quad l \in \mathbb{Z}, m \in \mathbb{N}^{*},
$$

then every $u \in \mathcal{H}$ has a Fourier series

$$
u=\sum_{k=1}^{n} \sum_{(l, m) \in \mathbb{Z} \times \mathbb{N}^{*}} u_{k l m} v_{l m} c_{k},
$$

where the $u_{k l m}$ satisfy $u_{k l m}=\overline{u_{k,-l, m}}$ to make the series real. If we define

$$
\operatorname{dom} \mathcal{L}=\{u \in \mathcal{H}: u \text { is given by }(2.1)\}
$$

with

$$
\sum_{k=1}^{n} \sum_{(l, m) \in \mathbb{Z} \times \mathbb{N}^{*}}\left(m^{2}-l^{2}\right)^{2}\left|u_{k l m}\right|^{2}<+\infty
$$

and

$$
\mathcal{L}: \operatorname{dom} \mathcal{L} \subset \mathcal{H} \rightarrow \mathcal{H}, \quad u \mapsto \sum_{k=1}^{n} \sum_{(l, m) \in \mathbb{Z} \times \mathbb{N}^{*}}\left(m^{2}-l^{2}\right) u_{k l m} v_{l m} c_{k},
$$

it is easy to check that $\mathcal{L}$ is a self-adjoint operator such that

$\operatorname{ker} \mathcal{L}=\operatorname{span}\left\{\cos m t \sin m x c_{k}, \sin m t \sin m x c_{k} \mid m \in \mathbb{N}^{*}, 1 \leq k \leq n\right\}$,

$$
\begin{gathered}
\operatorname{im} \mathcal{L}=(\operatorname{ker} \mathcal{L})^{\perp}, \\
\text { spectrum } \sigma(\mathcal{L})=\left\{m^{2}-l^{2} \mid l \in \mathbb{Z}, m \in \mathbb{N}^{*}\right\} .
\end{gathered}
$$

Moreover, for every $h \in \mathcal{H}, u$ is a GPDS on $\mathcal{J}$ of the system

$$
u_{t t}-u_{x x}=h
$$

if and only if $u \in \operatorname{dom} \mathcal{L}$ and $\mathcal{L} u=h$ (see [6] and references therein). Therefore, if we assume the existence of a constant $C \geq 0$ such that, for all $u \in \mathbb{R}^{n}$,

$$
\left\|V^{\prime \prime}(t, x, u)\right\| \leq C,
$$

it is well known that the mapping $N$ defined on $\mathcal{H}$ by

$$
(N(u))(t, x)=-V^{\prime}(t, x, u), \quad \text { a.e. on } \mathcal{J},
$$

continuously maps $\mathcal{H}$ into itself, and so the existence of GPDS on $\mathcal{J}$ for (1.1) is equivalent to the existence of a solution $u \in \operatorname{dom} \mathcal{L}$ for the equation

$$
\mathcal{L} u+N(u)+F(u)=h
$$

in $\mathcal{H}$, where the perturbing term $F: \operatorname{dom} \mathcal{L} \rightarrow \mathcal{H}$ is defined by

$$
(F(u))(t, x)=F(t, x, u), \quad \forall(t, x) \in \mathcal{J} .
$$


In the sequel, $\mathcal{B}$ will be the set of all continuous and nondecreasing mappings $\omega$ that satisfy

$$
\omega: \mathbb{R}_{+} \rightarrow \mathbb{R}_{+}, \omega(t)>0, t>0, \quad \int_{0}^{\infty} \frac{1}{\omega(t)} d t=\infty .
$$

Lemma 2.1 (see [8,9]). Assume that $\mathcal{H}$ is a Hilbert space. Let $\mathcal{T} \in C^{1}(\mathcal{H}, \mathcal{H})$, and assume that $\mathcal{T}^{\prime}(u)$ is everywhere invertible for all $u \in \mathcal{H}$. Then $\mathcal{T}$ is a global diffeomorphism onto $\mathcal{H}$ if there exists $\omega \in \mathcal{B}$ satisfying $\left\|\mathcal{T}^{\prime}(u)^{-1}\right\| \leq \omega(\|u\|)$.

\section{Existence and uniqueness}

Consider the boundary value problem (1.1). As shown in Section 2, if (2.4) holds, then (1.1) is equivalent to the operator equation

$$
\mathcal{L} u+N(u)+F(u)=h, \quad u \in \operatorname{dom} \mathcal{L} .
$$

Let $Q(u)=\left(V^{\prime \prime}(t, x, u)\right)$. Then

$$
\left(N^{\prime}(u) v\right)(t, x)=-\left(V^{\prime \prime}(t, x, u)\right) v(t, x)=-Q(t, x, u) v(t, x), \quad u, v \in \operatorname{dom}, L
$$

and $\mathcal{L}+N^{\prime}(u)=\mathcal{L}-Q(t, x, u)$, where $Q(u)$ is a symmetric matrix.

Let $b_{1}(t, x, u), \ldots, b_{n}(t, x, u)$ be eigenvalues of $Q(t, x, u)$, and for all $u \in$ $\operatorname{dom} L$,

$$
\alpha_{i}<b_{i}(t, x, u)<\beta_{i}, \quad i=1, \ldots, n,
$$

which shows that (2.4) holds, that is, there exists a constant $C$ such that $\left\|N^{\prime}(u) v\right\| \leq$ $C\|v\|$, for all $u, v \in \operatorname{dom} \mathcal{L}$.

For each fixed point $(t, x) \in \mathcal{J}$, consider the eigenvalue problem

$$
\mathcal{L} u-Q\left(t, x, u_{0}\right) u=\gamma u,
$$

where $u_{0} \in \operatorname{dom} \mathcal{L}$ is fixed. Since $\alpha_{i}, \beta_{i}, i=1, \ldots, n$, are consecutive and (3.1) holds, it follows that the eigenvalues of $Q\left(t, x, u_{0}\right)$ are ordered according to

$$
b_{1}\left(t, x, u_{0}\right) \leq b_{2}\left(t, x, u_{0}\right) \leq \cdots \leq b_{n}\left(t, x, u_{0}\right),
$$

and zero is not an eigenvalue of (3.2). Hence, $\mathcal{L}-Q\left(t, x, u_{0}\right)$ is invertible at $u_{0}$ for each fixed point $(t, x) \in \mathcal{J}$, and by the spectral theorem $[3,10,11]$

$$
\begin{aligned}
\left\|\left(\mathcal{L}-Q\left(t, x, u_{0}\right)\right)^{-1}\right\| & =\left\{\text { distance of } 0 \text { from the spectrum of } \mathcal{L}-Q\left(t, x, u_{0}\right)\right\}^{-1} \\
& \leq\left(\min _{1 \leq i \leq n}\left\{b_{i}\left(t, x, u_{0}\right)-\alpha_{i}, \beta_{i}-b_{i}\left(t, x, u_{0}\right)\right\}\right)^{-1}
\end{aligned}
$$

Let $\delta: \mathbb{R}_{+} \rightarrow \mathbb{R}_{+} \backslash\{0\}$ be defined by

$$
\delta(s)=\max _{\|u\| \leq s,(t, x) \in \mathcal{J}}\left\{\left(\min _{1 \leq i \leq n}\left\{b_{i}(t, x, u)-\alpha_{i}, \beta_{i}-b_{i}(t, x, u)\right\}\right)^{-1}\right\} .
$$

Then $\delta$ is continuous and nondecreasing with respect to $s$. Now since $u_{0}$ is arbitrary, we have that $\mathcal{L}+N^{\prime}(u)$ is invertible on $\mathcal{J}$ for all $u \in D(\mathcal{L})$, and $\left\|\left(\mathcal{L}+N^{\prime}(u)\right)^{-1}\right\| \leq$ $\delta(\|u\|)$. 
LEMMA 3.1. Assume that there exists $\eta<1$ with

$$
\left\|F_{u}(t, x, u)\right\| \leq \eta(\delta(\|u\|))^{-1} .
$$

Then

$$
\left\|\left[\mathcal{L}+N^{\prime}(u)+F^{\prime}(u)\right]^{-1}\right\| \leq \frac{\delta(s)}{1-\eta} .
$$

ProOf. From $\left(F^{\prime}(u) v\right)(t, x)=\left(F_{u}\right) v(t, x)$, for all $u, v \in \operatorname{dom} \mathcal{L}$,

$$
\left\|F^{\prime}(u) v\right\| \leq \eta(\delta(\|u\|))^{-1}\|v\| .
$$

For all $y \in \mathcal{H}$, notice that

$$
\left\|\left[\mathcal{L}+N^{\prime}(u)\right]^{-1} y\right\| \leq \delta(\|u\|)\|y\| .
$$

Define the mapping $P=F^{\prime}(u)\left[\mathcal{L}+N^{\prime}(u)\right]^{-1}: \mathcal{H} \rightarrow \mathcal{H}$. Then from (3.7) and (3.8), for all $y \in \mathcal{H}$,

$$
\begin{aligned}
\|P y\| & =\left\|F^{\prime}(u)\left[\mathcal{L}+N^{\prime}(u)\right]^{-1} y\right\| \\
& \leq \eta(\delta(\|u\|))^{-1}\left\|\left[\mathcal{L}+N^{\prime}(u)\right]^{-1} y\right\| \\
& \leq \eta(\delta(\|u\|))^{-1} \delta(\|u\|)\|y\|=\eta\|y\| .
\end{aligned}
$$

Then $I+P$ is invertible and $\left\|[I+P]^{-1}\right\| \leq(1-\eta)^{-1}$. Note that

$$
\begin{aligned}
\mathcal{L}+N^{\prime}(u)+F^{\prime}(u) & =\left(I+F^{\prime}(u)\left[\mathcal{L}+N^{\prime}(u)\right]^{-1}\right) \cdot\left(\mathcal{L}+N^{\prime}(u)\right) \\
& =(I+P) \cdot\left(\mathcal{L}+N^{\prime}(u)\right) .
\end{aligned}
$$

Hence, it follows from the invertibility of $I+P$ that $\mathcal{L}+N^{\prime}(u)+F^{\prime}(u)$ is invertible and $\left[\mathcal{L}+N^{\prime}(u)+F^{\prime}(u)\right]^{-1}=\left[\mathcal{L}+N^{\prime}(u)\right]^{-1}(I+P)^{-1}$. This, together with (3.4), yields (3.6).

THEOREM 3.2. Assume that (A1) and (3.5) hold. Then (1.1) with the periodicDirichlet boundary conditions on $\mathcal{J}$ has a unique generalized solution $u \in\left(L^{2}(\mathcal{J})\right)^{n}$ for every $h \in\left(L^{2}(\mathcal{J})\right)^{n}$.

PROOF. From (3.4),

$$
\begin{aligned}
\delta(s) & =\max _{\|u\| \leq s,(t, x) \in \mathcal{J}}\left\{\left(\min _{1 \leq i \leq n}\left\{b_{i}(t, x, u)-\alpha_{i}, \beta_{i}-b_{i}(t, x, u)\right\}\right)^{-1}\right\} \\
& \leq \max _{\|u\| \leq s,(t, x) \in \mathcal{J}}\left\{\left(\min _{1 \leq i \leq n}\left\{\alpha_{i}+\phi_{i}(t, x,\|u\|)-\alpha_{i}, \beta_{i}-\beta_{i}+\varphi_{i}(t, x,\|u\|)\right\}\right)^{-1}\right\} \\
& =\max _{\|u\| \leq s,(t, x) \in \mathcal{J}}\left\{\left(\min _{1 \leq i \leq n}\left\{\phi_{i}(t, x,\|u\|), \varphi_{i}(t, x,\|u\|)\right\}\right)^{-1}\right\} .
\end{aligned}
$$

Thus

$$
\begin{aligned}
\int_{0}^{\infty} \frac{1}{\delta(s)} d s & \geq \int_{0}^{\infty}\left(\max _{\|u\| \leq s,(t, x) \in \mathcal{J}}\left\{\left(\min _{1 \leq i \leq n}\left\{\phi_{i}(t, x,\|u\|), \varphi_{i}(t, x,\|u\|)\right\}\right)^{-1}\right\}\right)^{-1} d s \\
& \geq \int_{0}^{\infty} \min _{1 \leq i \leq n,(t, x) \in \mathcal{J}}\left\{\phi_{i}(t, x,\|s\|), \varphi_{i}(t, x,\|s\|)\right\} d s .
\end{aligned}
$$


Then, by (1.5) in assumption (A1), Lemma 2.1 (with (3.6)) and Lemma 3.1, the system (1.1) has a unique generalized solution $u \in\left(L^{2}(\mathcal{J})\right)^{n}$ for every $h \in\left(L^{2}(\mathcal{J})\right)^{n}$. The proof is complete.

We now use the following assumption.

(A2). There exist two symmetric $n \times n$ matrices $A$ and $B$ such that

$$
A+\phi(t, x,\|u\|) I \leq V^{\prime \prime} \leq B-\varphi(t, x,\|u\|) I
$$

on $\mathcal{J} \times \mathbb{R}^{n}$, and the eigenvalues of $A$ and $B$ are $\alpha_{i}, \beta_{i}, i=1, \ldots, n$, respectively, where $I$ is the $n \times n$ identity matrix, $\phi_{i}(t, x, s)$ and $\varphi_{i}(t, x, s), i=1, \ldots, n$, are continuous functions defined from $\mathcal{J} \times[0, \infty)$ to $(0, \infty)$ that are nonincreasing with respect to $s$, and

$$
\int_{0}^{+\infty} \min _{(t, x) \in \mathcal{J}}\{\phi(t, x, s), \varphi(t, x, s)\} d s=+\infty .
$$

Here $\alpha_{i}, \beta_{i} \in \sigma(L), i=1, \ldots, n$, are consecutive.

Essentially the same reasoning as in Theorem 3.2 yields the following result.

TheORem 3.3. Assume that (A2) and (3.5) hold. Then (1.1) with the periodicDirichlet boundary conditions on $\mathcal{J}$ has a unique generalized solution $u \in\left(L^{2}(\mathcal{J})\right)^{n}$ for every $h \in\left(L^{2}(\mathcal{J})\right)^{n}$.

REMARK 3.4. Theorems 3.2 and 3.3 allow the eigenvalues of $V^{\prime \prime}(t, x, u)$, when $\|u\| \rightarrow \infty$, to interact with points of the spectral set $\left\{m^{2}-l^{2} \mid l \in \mathbb{Z}, m \in \mathbb{N}^{*}\right\}$. Consider the nonlinear semilinear-wave equation

$$
u_{t t}-u_{x x}+V^{\prime}(t, x, u)=h(t, x), \quad \forall(t, x) \in \mathcal{J},
$$

with the periodic-Dirichlet boundary conditions on $\mathcal{J}$. Let

$$
V^{\prime}(t, x, u)=m u-\frac{\sin ^{2}(t)+1}{4} \ln \left(u+\sqrt{1+u^{2}}\right), \quad m \in\{1,2, \ldots\},
$$

and let $h: \mathcal{J} \rightarrow \mathbb{R}$ be in $L^{2}(\mathcal{J})$. Theorem 3.2 guarantees the existence of a unique periodic-Dirichlet solution to (3.10) since

$$
m-1+\frac{1}{2} \leq V^{\prime \prime}(t, x, u)=m-\frac{\sin ^{2}(t)+1}{4 \sqrt{1+u^{2}}} \leq m .
$$

Also,

$$
\lim _{\|u\| \rightarrow \infty}\left\|V^{\prime \prime}(t, x, u)-m\right\|=0 .
$$

We now discuss the case where the eigenvalues of $V^{\prime \prime}(t, x, u)$ do not interact with points of the spectral set $\left\{m^{2}-l^{2} \mid l \in \mathbb{Z}, m \in \mathbb{N}^{*}\right\}$ as $\|u\| \rightarrow \infty$. 
Corollary 3.5. Suppose that

$$
A_{1} \leq V^{\prime \prime} \leq B_{1}, \quad \alpha_{i}<\mu_{i} \leq \nu_{i}<\beta_{i},
$$

where $\mu_{i}$ and $v_{i}$ are eigenvalues of the symmetric $n \times n$ matrices $A_{1}$ and $B_{1}$, respectively, and $\alpha_{i}, \beta_{i} \in \sigma(\mathcal{L}), i=1, \ldots, n$, are consecutive. Assume that (3.5) holds. Then (1.1) with the periodic-Dirichlet boundary conditions on $\mathcal{J}$ has a unique generalized solution $u \in\left(L^{2}(\mathcal{J})\right)^{n}$ for every $h \in\left(L^{2}(\mathcal{J})\right)^{n}$.

PROOF. It follows from (3.11) that the eigenvalues $\lambda_{i}, i=1, \ldots, n$, of $V^{\prime \prime}$ satisfy

$$
\alpha_{i}+\min _{1 \leq i \leq n}\left(\mu_{i}-\alpha_{i}\right) \leq \lambda_{i}\left(V^{\prime \prime}\right) \leq \beta_{i}-\min _{1 \leq i \leq n}\left(\beta_{i}-v_{i}\right)
$$

If we let $\phi_{j}(t, x, s)=\min _{1 \leq i \leq n}\left(\mu_{i}-\alpha_{i}\right), \varphi_{j}(t, x, s)=\min _{1 \leq i \leq n}\left(\beta_{i}-v_{i}\right), j=$ $1, \ldots, n$, then (1.5) holds. The result follows from Theorem 3.2.

REMARK 3.6. Since $\alpha_{i}, \beta_{i} \in \sigma(\mathcal{L}), i=1, \ldots, n$, are consecutive in Corollary 3.5, the respective eigenvalues $\mu_{1} \leq \mu_{2} \cdots \leq \mu_{n}$ and $v_{1} \leq v_{2} \cdots \leq v_{n}$ of $A_{1}$ and $B_{1}$ satisfy

$$
\bigcup_{k=1}^{n}\left[\mu_{i}, v_{i}\right] \cap\left\{m^{2}-l^{2} \mid l \in \mathbb{Z}, m \in \mathbb{N}^{*}\right\}=\varnothing .
$$

Then Theorem 1.1, that is, the main result of [6], is a special case of Corollary 3.5, when the perturbing term $F(t, x, u)=0$.

\section{References}

[1] P. W. Bates, 'Solutions of nonlinear elliptic systems with meshed spectra', Nonlinear Anal. 4 (1980), 1023-1030.

[2] P. W. Bates and A. Castro, 'Existence and uniqueness for a variational hyperbolic system without resonance', Nonlinear Anal. 4 (1980), 1151-1156.

[3] N. Dunford and J. T. Schwartz, Linear Operators Part II: Spectral Theory, Self Adjoint Operators in Hilbert Space (Wiley Interscience, New York, 1988).

[4] A. C. Lazer, 'Application of a lemma on bilinear forms to a problem in nonlinear oscillations', Proc. Amer. Math. Soc. 33 (1972), 89-94.

[5] J. Mawhin, 'Contractive mappings and periodically perturbed conservative systems', Arch. Math. (Brno) 12 (1976), 67-74.

[6] J. Mawhin, 'Conservative systems of semilinear wave equations with periodic-Dirichlet boundary conditions', J. Differential Equations 42 (1981), 116-128.

[7] J. Mawhin and J. R. Ward Jr, 'Asymptotic nonuniform nonresonance conditions in the periodicDirichlet problem for semilinear wave equations', Ann. Mat. Pura Appl. 135 (1983), 85-97.

[8] R. Plastock, 'Homeomorphisms between Banach spaces', Trans. Amer. Math. Soc. 200 (1974), 169-183.

[9] M. Radulescu and S. Radulescu, 'Global inversion theorems and applications to differential equations', Nonlinear Anal. 4 (1980), 951-965.

[10] Z. H. Shen, 'On the periodic solution to the Newtonian equation of motion', Nonlinear Anal. 13 (1989), 145-149.

[11] Z. H. Shen and M. A. Wolfe, 'On the existence of periodic solutions of periodically perturbed conservative systems', J. Math. Anal. Appl. 153 (1990), 78-83. 
JINHAI CHEN, Department of Mathematical and Statistical Sciences, University of Colorado Denver, Campus Box 170, PO Box 173364, Denver, CO 80217-3364, USA e-mail: cjh_maths@yahoo.com.cn

DONAL O'REGAN, Department of Mathematics, National University of Ireland, Galway, Ireland

e-mail: donal.oregan@nuigalway.ie 